tion which nurture and support the practicing physician. The AAFP is the center of the family and leads in advocacy that affects us all.

So what does this mean for you? It boils down to participation and engagement. Many family physicians are members of 2 or 3 of these organizations. Are you looking for ways to be part of this important work? More than ever each organization needs your support, participation in advocacy, and your ideas for solutions. Find your niche, join a task force or committee, step into a leadership role, and find a seat at the table. Your family needs you-now.

Michael Tuggy, $M D_{i}$ Michael Mazzone, $M D$ Stoney Abercrombie, $M D_{i}$ Brian Crownover, $M D_{\text {; }}$ Grant Hoekzema, MD, Nathan Krug, MDi Lisa Maxwell, MD, Karen Mitchell, MD, Stephen Scbultz, MD, Todd Shaffer, MD, MBA

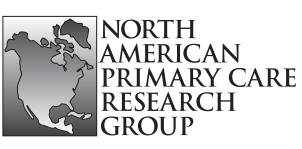

From the North American Primary Care Research Group

Ann Fam Med 2013;386-387. doi:10.1370/afm.1559.

\section{PRIMARY CARE RESEARCH FROM THE WOMEN'S HEALTH INITIATIVE}

Primary care research can encompass not only a broad range of topics, but also a variety of methodologies, one being secondary data analysis. Secondary data analysis involves the analysis of existing data to evaluate questions not addressed by the original study, and can be used by primary care researchers to conduct clinical, epidemiological, and health services research. The use of secondary data analysis can have several advantages, as it can provide access to large sample sizes in a quick and inexpensive manner. However, the data available is often limited by the measures included in the original study. A variety of sources for secondary data are available, such as the Women's Health Initiative. The Women's Health Initiative is an ongoing study of a multiethnic cohort of postmenopausal women from 40 centers in the United States. This study is in its second extension and has over 15 years of cumulative data. Its present focus is on healthy aging, natural history of multiple chronic diseases, symptoms, and functional status. It is thus an ideal cohort for primary care researchers interested in these outcomes. One of the distinguished papers presented at the 40th NAPCRG Annual Meeting in the fall of 2012, reported successfully performed secondary data analysis of the Women's Health Initiative. The distin- guished paper, entitled Social Support and Physical Activity as Moderators of Life Stress in Predicting Baseline Depression and Change in Depression Over Time in the Women's Health Initiative, was presented by primary care researcher Lisa Uebelacker, $\mathrm{PhD}$, from Brown University.

Uebelacker's research expands on previous research that has shown negative life events, acute stressors and chronic stressors increase risk for onset, persistence, or worsening of depression. Different types of stressors may increase risk for depression. These include interpersonal stressors, such as verbal abuse, physical abuse, social strain, care giving, or negative interpersonal life events ${ }_{i}$ financial stressors such as low income or selfreport of financial stress; and medical stressors such as chronic medical conditions or pain. In contrast, social support and physical activity may decrease the risk of depression. The purpose of this analysis was to determine whether social support and/or physical activity actually buffer the association between stressors and increased risk of depression symptoms at a single time point and after a 3-year follow-up period.

Uebelacker and colleagues conducted a secondary analysis of data from the Women's Health Initiative Observational Study. This prospective cohort includes 92,063 community-dwelling post-menopausal women who participated in the study. Depression symptoms were measured at baseline and 3 years later, social support, physical activity, and stressors were measured at baseline. For baseline analyses, the investigators used the entire sample; in order to look at new-onset depression at 3-year follow-up, they used data only from the 68,368 women who were not depressed at baseline and provided follow-up data. They conducted adjusted logistic regressions, with depression status as the dependent variable.

Stressors at baseline, including verbal abuse, physical abuse, care giving, social strain, negative life events, financial stress, low income, acute pain, and a greater number of chronic conditions, were all associated with higher levels of depression symptoms at baseline and new onset elevated symptoms at 3 -year follow-up. Social support and physical activity were associated with lower levels of depressive symptoms at baseline and at 3-year follow-up. Social support at baseline attenuated the association between concurrent depression and physical abuse, number of medical conditions, financial stress, social strain, and low income. Social support also attenuated the association between financial stress and low income on new-onset depression 3 years later. Physical activity was not a significant effect moderator.

These results highlight the important role that social support can play in reducing risk of depression in older women, particularly in times of stress. Although physical activity did not buffer the effects 
of stress on depression, investigators did find a main effect for physical activity, such that those women with increased physical activity were less likely to develop high levels of depression symptoms 3 years later.

Data from the Women's Health Initiative (WHI) is available for review at the WHI website: http://www. whi.org. Ancillary study proposals, joining working groups, writing groups, and new paper proposals are actively supported by principal investigators at most of the 40 sites. Dr. Charles B. Eaton, senior author on the above paper, is the Principal Investigator at the Brown University, Pawtucket WHI site, and is glad to support any NAPCRG affiliated investigator interested in the WHI datasets. E-mail: charles_eaton@mhri.org.

Charles Eaton, MD, MS

Professor of Family Medicine and Epidemiology Alpert Medical School of Brown University Director, Center for Primary Care and Prevention

\section{Prom the American Academy}

Ann Fam Med 2013;387-388. doi:10.1370/afm.1560.

AAFP INTERVIEW WITH FARZAD
MOSTASHARI, MD, MS, NATIONAL
COORDINATOR FOR HEALTH
INFORMATION TECHNOLOGY

AAFP News Now recently sat down with Farzad Mostashari, MD, MS, head of the Office of the National Coordinator (ONC) for Health Information Technology (IT). This office is responsible for rolling out the various health IT incentives and penalties contained in the Health Information Technology for Economic and Clinical Health (HITECH) Act, which was enacted as part of the 2009 American Recovery and Reinvestment Act.

Many physicians who have adopted health IT in the form of electronic health records (EHRs) have expressed disappointment with the technology as it currently exists, so we asked Mostashari some of the questions family physicians have been asking the AAFP.

Q Physicians were told at the beginning of the EHR era that after the hard work of implementation, they would see the value of their investment in terms of gained productivity. How close are we to achieving that promise?

\footnotetext{
A If physicians just replicate the existing paper-based processes in a digital way, they probably are not going
} to get huge productivity gains. But if they redesign the care flow to designate what things are done by people versus what's done through the EHR technology, then that really adds to productivity. That's how I would summarize the experience of folks who have made EHR implementation a wonderful business decision.

It's important for physicians who have found productivity gains and value in their EHRs to share their stories. We know that successful implementation can be done, and is being done, by many excellent physicians who were able to-and I think this is the key-change their processes to take full advantage of their EHRs.

Q Many physicians are not seeing the expected financial return on investment after EHR implementation. Why is that?

A How you implement the technology has a lot to do with the results you achieve. But the bigger issue is how the compensation system is designed. If physicians are operating in a fee-for-service environment, then many of the gains of EHRs - for instance, in quality, safety and patient engagement—aren't reflected in revenue. Physicians are doing more work and delivering better care and service, but the added value is not reflected in the reimbursement.

We've been an advocate for making sure that when value is added, it's reflected in increased physician reimbursement whether it's through the patient-centered medical home $(\mathrm{PCMH})$ setting, value-based purchasing or part of an accountable care structure. That's where the ability to manage information - not just for individual patients but for populations-becomes an absolute necessity, because in those models, it's not a question of whether there's a return on investment with electronic health records. A physician can't function in those models without an EHR.

Q Some recent research on EHRs has suggested that technology does not always improve patient care. Any ideas on what's holding back progress?
A If you look at different studies, you'll find some vari- ance in terms of results. There are two things to pay attention to here. First, what does the bulk of the evidence say? If you actually do an evidence-based review of the lit- erature-and we asked the RAND Corp. to do that for us- you find that upward of $80 \%$ of all studies on EHRs show positive results. So the evidence is there, but clearly there is a perception that the technology isn't helping physicians improve care.

That brings me to the second point. I think we have a natural tendency to focus on things that are counterintuitive. For instance, Kaiser (Family Foundation) published a study with 100,000 patients with diabetes and found that their care was dramatically improved with EHRs. That study didn't get much column space. But a small study (conducted by another researcher) that was focused on one setting with one particular EHR found there was no improvement in care quality. That study got a lot of ink. 